\title{
Mathematical modeling of the impact of forest fires on buildings and structures
}

\author{
Valeriy Perminov*, and Victoria Marzaeva \\ Department of Control and Diagnostics, Tomsk Polytechnic University, 634045 Tomsk, Russia
}

\begin{abstract}
The protection of buildings and structures in a community from destruction by forest fires is a very important concern. This paper addresses the development of a mathematical model for fires in the wildland-urban intermix. The forest fire is a very complicated phenomenon. At present, fire services can forecast the danger rating of, or the specific weather elements relating to, forest fire. There is need to understand and predict forest fire initiation, behavior and impact of fire on the buildings and constructions. This paper's purposes are the improvement of knowledge on the fundamental physical mechanisms that control forest fire behavior. The mathematical modeling of forest fires actions on buildings and structures has been carried out to study the effects of fire intensity and wind speed on possibility of ignition of buildings.
\end{abstract}

\section{Mathematical model}

A great deal of work has been done on the theoretical problem of forest fires [1-4]. However, the study of crown fires initiation and spread $[9,10]$ has been limited mainly to cases studied of forest fires propagation without take into account the mutual interaction of crown forest fires with different obstacles (roads, glades and etc.), buildings and constructions. In this paper, the impacts of crown forest fires on building are studied. The dangerous distances between forest and buildings are calculated in cases when the buildings will be ignited under the influence of forest fires. It is assumed that the forest during a forest fire can be modeled as 1) a multi-phase, multistoried, spatially heterogeneous medium; 2) in the fire zone the forest is a porous-dispersed, two-temperature, singlevelocity, reactive medium; 3 ) the forest canopy is supposed to be non - deformed medium (trunks, large branches, small twigs and needles), which affects only the magnitude of the force of resistance in the equation of conservation of momentum in the gas phase, i.e., the medium is assumed to be quasi-solid (almost non-deformable during wind gusts); 4) let there be a so-called "ventilated" forest massif, in which the volume of fractions of condensed forest fuel phases, consisting of dry organic matter, water in liquid state, solid pyrolysis products, and ash, can be neglected compared to the volume fraction of gas phase (components of air and gaseous pyrolysis products); 5) the flow has a developed turbulent nature and molecular transfer is neglected; 6) gaseous phase density doesn't depend on the pressure because of the low velocities of the flow in comparison with the velocity of the

\footnotetext{
* Corresponding author: perminov@tpu.ru
} 
sound. The modeling approach is based on the use of standard non-stationary threedimensional conservation. The problem formulated above reduces to the solution of the next system of equations:

$$
\begin{aligned}
& \frac{\partial \rho}{\partial t}+\frac{\partial}{\partial x_{j}}\left(\rho v_{j}\right)=Q, j=\overline{1,3}, i=\overline{1,3} \\
& \rho \frac{d v_{i}}{d t}=-\frac{\partial P}{\partial x_{i}}+\frac{\partial}{\partial x_{j}}\left(-\rho{\overline{v_{i}^{\prime}}}_{j}^{\prime}\right)-\rho s c_{d} v_{i}|\vec{v}|-\rho g_{i}-Q v_{i} ; \\
& \rho c_{p} \frac{d T}{d t}=\frac{\partial}{\partial x_{j}}\left(-\rho c_{p} v_{j}^{\prime} \overline{\left.T^{\prime}\right)}+q_{5} R_{5}-\alpha_{v}\left(T-T_{s}\right)+k_{g}\left(c U_{R}-4 \sigma T^{4}\right) ;\right. \\
& \rho \frac{d c_{\alpha}}{d t}=\frac{\partial}{\partial x_{j}}\left(-\rho \overline{v_{j}^{\prime} c_{\alpha}^{\prime}}\right)+R_{5 \alpha}-Q c_{\alpha}, \alpha=1,2 ; \\
& \frac{\partial}{\partial x_{j}}\left(\frac{c}{3 k} \frac{\partial U{ }_{R}}{\partial x_{j}}\right)-k c U{ }_{R}+4 k_{S} \sigma T_{S}^{4}+4 k_{g} \sigma T^{4}=0, \\
& k=k_{g}+k_{S} ; \\
& \sum_{i=1}^{4} \rho_{i} c_{p i} \varphi_{i} \frac{\partial T_{S}}{\partial t}==q_{3} R_{3}-q_{2} R_{2}-k_{S}\left(c U_{R}-4 \sigma T_{S}^{4}\right)+\alpha_{v}\left(T-T_{S}\right) ; \\
& \rho_{1} \frac{\partial \varphi_{1}}{\partial t}=-R_{1 s}, \rho_{2} \frac{\partial \varphi_{2}}{\partial t}=-R_{2 s}, \rho_{3} \frac{\partial \varphi_{3}}{\partial t}=\alpha_{C} R_{1 s}-\frac{M_{C}}{M_{1}} R_{3 w}, \rho_{4} \frac{\partial \varphi_{4}}{\partial t}=0 ; \\
& \sum_{\alpha=1}^{3} c_{\alpha}=1, P_{e}=\rho R T \sum_{\alpha=1}^{3} \frac{c_{\alpha}}{M_{\alpha}}, \vec{v}=\left(v_{1}, v_{2}, v_{3}\right), \vec{g}=(0,0, g) .
\end{aligned}
$$

The system of equations (1)-(7) must be solved taking into account the initial and boundary conditions:

$$
\begin{gathered}
t=0: v_{1}=0, v_{2}=0, v_{3}=0, T=T_{e}, c_{\alpha}=c_{\alpha e}, T_{s}=T_{s e}, \varphi_{i}=\varphi_{i e} ; \\
x_{1}=0: v_{1}=V, v_{2}=0, v_{3}=0, T=T_{e}, c_{\alpha}=c_{\alpha e},-\frac{c}{3 k} \frac{\partial U_{R}}{\partial x_{1}}+\frac{c}{2} U_{R}=0 ; \\
x_{1}=x_{1 e}: \frac{\partial v_{1}}{\partial x_{1}}=0, \frac{\partial v_{2}}{\partial x_{1}}=0, \frac{\partial v_{3}}{\partial x_{1}}=0, \frac{\partial T}{\partial x_{1}}=0, \frac{\partial c_{\alpha}}{\partial x_{1}}=0, \frac{c}{3 k} \frac{\partial U_{R}}{\partial x_{1}}+\frac{c}{2} U_{R}=0 ; \\
x_{2}=-x_{2 e}: \frac{\partial v_{1}}{\partial x_{2}}=0, \frac{\partial v_{2}}{\partial x_{2}}=0, \frac{\partial v_{3}}{\partial x_{2}}=0, \frac{\partial T}{\partial x_{2}}=0, \frac{\partial c_{\alpha}}{\partial x_{2}}=0,-\frac{c}{3 k} \frac{\partial U_{R}}{\partial x_{2}}+\frac{c}{2} U_{R}=0 ; \\
x_{2}=x_{2 e}: \frac{\partial v_{1}}{\partial x_{2}}=0, \frac{\partial v_{2}}{\partial x_{2}}=0, \frac{\partial v_{3}}{\partial x_{2}}=0, \frac{\partial T}{\partial x_{2}}=0, \frac{\partial c_{\alpha}}{\partial x_{2}}=0, \frac{c}{3 k} \frac{\partial U_{R}}{\partial x_{2}}+\frac{c}{2} U_{R}=0 ; \\
x_{3}=0: v_{1}=0, v_{2}=0, \frac{\partial c_{\alpha}}{\partial x_{3}}=0,-\frac{c}{3 k} \frac{\partial U_{R}}{\partial x_{3}}+\frac{c}{2} U_{R}=0, \\
\rho v_{3}=\rho_{0} \omega_{0}, T=T_{0},\left|x_{1}\right| \leq x_{0},\left|x_{2}\right| \leq x_{0}, \\
\rho v_{3}=0, T=T_{e},\left|x_{1}\right|>x_{0},\left|x_{2}\right|>x_{0} ; \\
x_{3}=x_{3 e}: \frac{\partial v_{1}}{\partial x_{3}}=0, \frac{\partial v_{2}}{\partial x_{3}}=0, \frac{\partial v_{3}}{\partial x_{3}}=0, \frac{\partial T}{\partial x_{3}}=0, \frac{\partial c_{\alpha}}{\partial x_{3}}=0, \frac{c}{3 k} \frac{\partial U_{R}}{\partial x_{3}}+\frac{c}{2} U_{R}=0 .
\end{gathered}
$$


Here and above $\frac{\mathrm{d}}{\mathrm{d} t}$ is the symbol of the total (substantial) derivative; $\alpha_{v}$ is the coefficient of phase exchange; $\rho$ - density of gas - dispersed phase, $t$ is time; $v_{i}$ - the velocity components; $T, T_{S}$, - temperatures of gas and solid phases, $U_{R}$ - density of radiation energy, $k$ - coefficient of radiation attenuation, $P$ - pressure; $c_{p}$ - constant pressure specific heat of the gas phase, $c_{p i}, \rho_{i}, \varphi_{1}$ - specific heat, density and volume of fraction of condensed phase ( 1 - dry organic substance, 2 - moisture, 3 - condensed pyrolysis products, 4 - mineral part of forest fuel), $R_{i}$ - the mass rates of chemical reactions, $q_{i}$ - thermal effects of chemical reactions; $k_{g}, k_{S}$ - radiation absorption coefficients for gas and condensed phases; $T_{e}$ - the ambient temperature; $c_{\alpha}$ - mass concentrations of $\alpha$ - component of gas - dispersed medium, index $\alpha=1,2,3$ where 1 corresponds to the density of oxygen, 2 - to carbon monoxide $C O, 3$ - to carbon dioxide and inert components of air; $R$ - universal gas constant; $M_{\alpha}, M_{C}$, and $M$ molecular mass of $\alpha$-components of the gas phase, carbon and air mixture; $g$ is the gravity acceleration; $c_{d}$ is an empirical coefficient of the resistance of the vegetation, $s$ is the specific surface of the forest fuel in the given forest stratum. To define source terms which characterize inflow (outflow of mass) in a volume unit of the gas-dispersed phase, the formulae were used for the rate of formulation of the gas-dispersed mixture $Q$, outflow of oxygen $R_{51}$, changing carbon monoxide $R_{52}$ [3]. It is supposed that the optical properties of a medium are independent of radiation wavelength (the assumption that the medium is "grey"), and the so-called diffusion approximation for radiation flux density were used for a mathematical description of radiation transport during forest fires. To close the system (1)(7), the components of the tensor of turbulent stresses, and the turbulent heat and mass fluxes are determined using the local-equilibrium model of turbulence [3]. It should be noted that this system of equations describes processes of transfer within the entire region of the forest massif, which includes the space between the underlying surface and the base of the forest canopy, the forest canopy and the space above it, while the appropriate components of the data base are used to calculate the specific properties of the various forest strata and the near-ground layer of atmosphere. This approach substantially simplifies the technology of solving problems of predicting the state of the medium in the fire zone numerically. The thermodynamic, thermophysical and structural characteristics correspond to the forest fuels in the canopy of a different (for example pine [3]) type of forest.

\section{Numerical solution and results}

The boundary-value problem (1)-(14) is solved numerically. A discrete analog was obtained by means of the control volume method using the SIMPLE algorithm (Patankar [5]). Difference equations that arise in the course of sampling were resolved by the method of SIP [5]. In order to efficiently solve this problem in a reactive flow the method of splitting according to physical processes was used. The accuracy of the program was checked by the method of inserted analytical solutions. The effect of the dimensions of the control volumes on the solution was studied by diminishing them. Fields of temperature, velocity, component mass fractions, and volume fractions of phases were obtained numerically in different instants of time. Fig.1. shows temperature fields at the different instants moments of forest fire spread for a wind speed of $15 \mathrm{~m} / \mathrm{s}(1-1.2,2-1.5,3-2 ., 4$ - 3., $\left.5-4 ; \bar{T}=\mathrm{T} / \mathrm{T}_{\mathrm{e}} \mathrm{T}_{\mathrm{e}}=300 \mathrm{~K}\right)$. The first stage is related to increasing maximum temperature in the place of ignition with the result that a crown fire source appears (Fig.1a). The results of the calculation give an opportunity to consider forest fire spread and distribution of temperature in domain for different instants of time. It is considered the 
effect of forest fire front on the building which is situated near from the forest on the right side. The results of calculations can be used to evaluate the thermal effects on the building. The temperature fields of crown forest fire at definite moment will be interacted with the obstacle - building (Figure $1 \mathrm{~b}$ )-d)) and can be ignited it. During this process, the surface of the wall of the building heats as a result of convection and radiation heat transfer. The wood building will be ignited at definite temperature. It depends on wind velocities, distances from the forest fire to building, the height of building and others parameters.
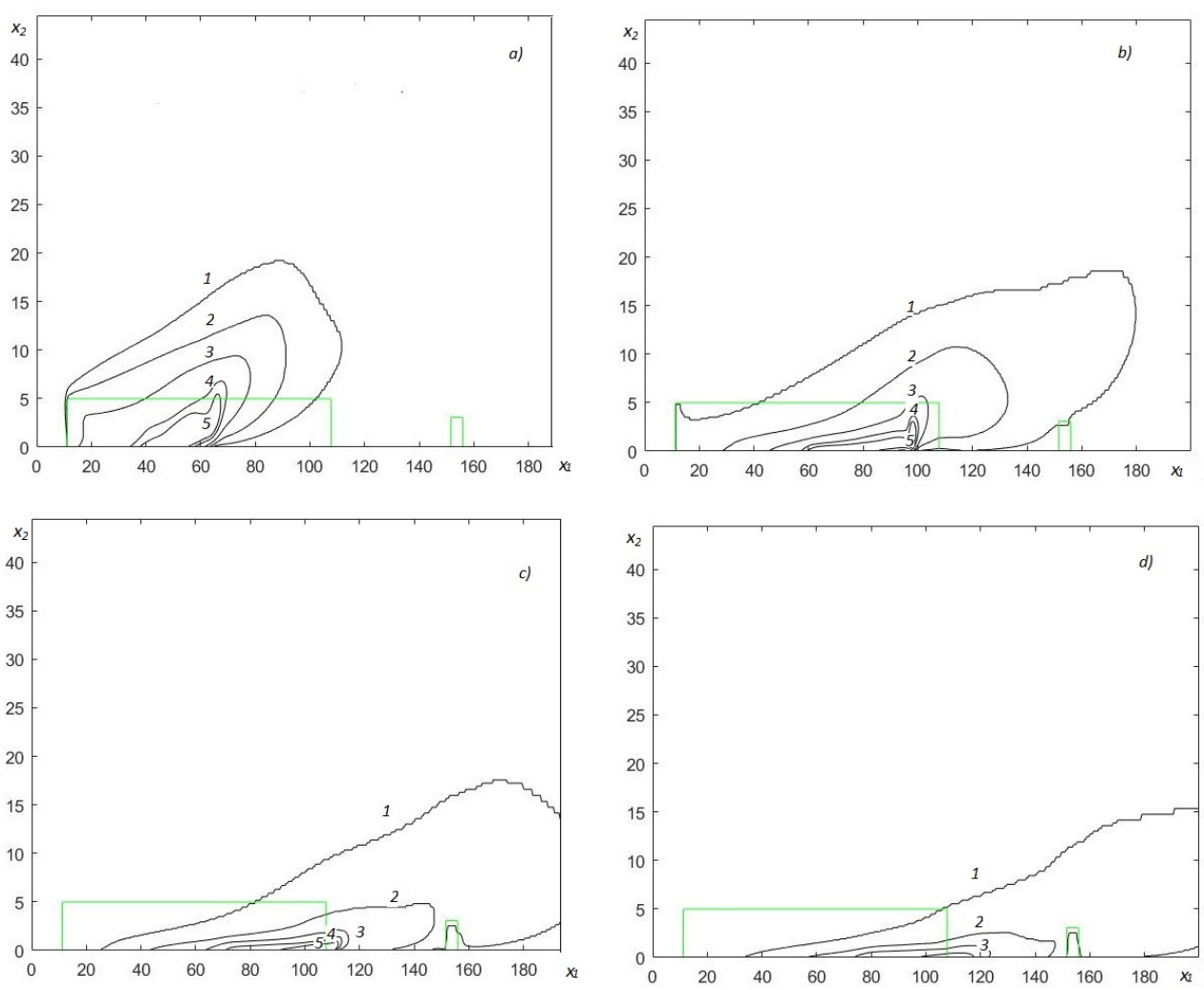

Fig. 1. Gas temperature field at a) $t=15 \mathrm{~s}, b) t=20 \mathrm{~s}, c) t=23 \mathrm{~s}, d) t=25 \mathrm{~s}$.

In paper [6] it is showed that the wood will be ignited when its temperature exceeds $300 \mathrm{C}$. As a result of the numerical calculations it was defined maximum safety distances between forest and building when the building would not have been ignited by forest fire (Fig. 2). The wind speed values increase from 6 to $14 \mathrm{~m} / \mathrm{s}$. Also, it was studied the influence of the height of building on the value of safety distances. When the height of building changes from 3 to $6 \mathrm{~m}$ (line 1 and 2 correspondently), the safety distances $l$ also increases (Figure 2) for different values of wind speed. 


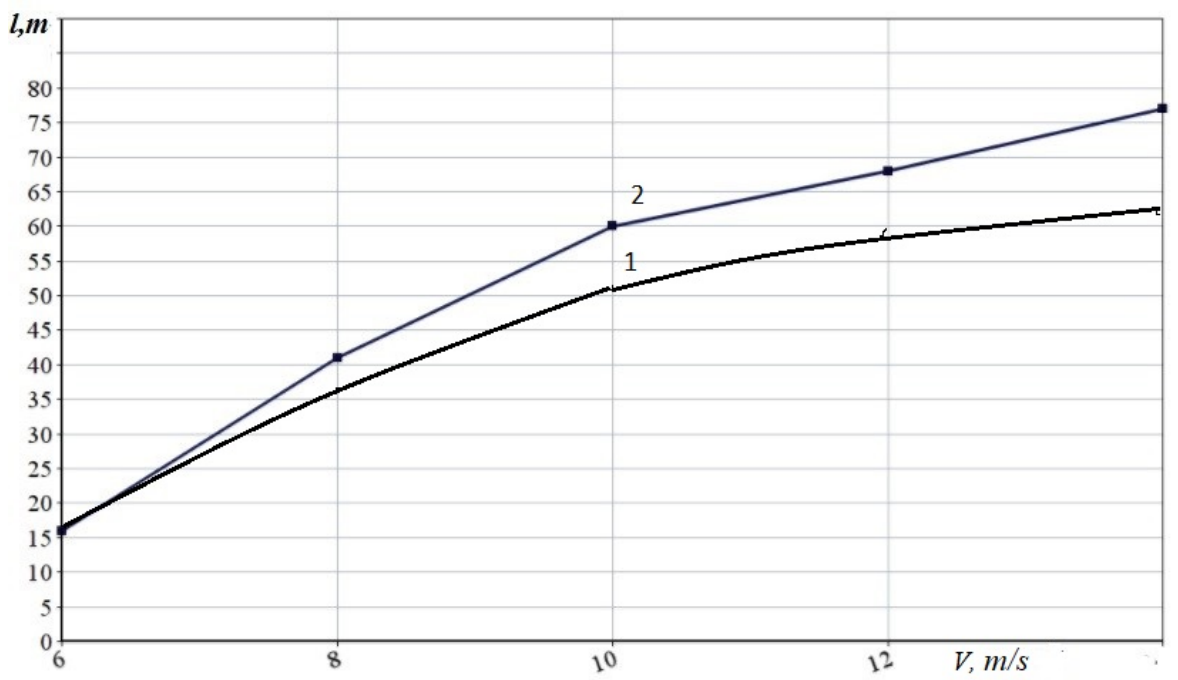

Fig. 2. The dependence of safety distances between forest and building as a function of wind speed values; $\mathrm{H}=3 \mathrm{~m}$ (1) and $\mathrm{H}=6 \mathrm{~m}(2)$.

A multiphase mathematical model of wind-aided crown forest fires propagating through heterogeneous fuel beds has been performed. It takes into account the hydrodynamic aspects of the flow and uses Arrhenius kinetics to describe the basic physics and chemical processes of thermal decomposition heating, drying, pyrolysis, and combustion. Turbulence and radiation are considered in order to improve the physical insight. It allows to investigate the dynamics of the impact of forest fires on buildings under the influence of various external conditions: a) meteorology conditions (air temperature, wind velocity etc.), b) type (various kinds of forest combustible materials) and their state (load, moisture etc.). The calculations let to get the maximum distance from the fire to the building in which the object possible ignition. It has been found that the effect of increasing the wind speed is to increase the safety distances between forest and building. The increasing of building height is observed also led to increase the safety distances between forest and building. Specific experiments are also needed to obtain more reliable information on validation of further solution of this problem.

The paper was supported from RFBR (project code: № 16-41-700022 p_a) and within the framework of Tomsk Polytechnic University Competitiveness Enhancement Program grant.

\section{References}

[1] R.C. Rothermal, Predicting behavior and size of crown fires in the Northern Rocky Mountains, Res.Pap. INT-438. Ogden, UT: US Department of Agriculture, Forest Service, Intermountain Forest and Range Experiment Station (1972)

[2] F.A. Albini, F.A. et al., Int. J. of Wildland Fire, 5 (1995)

[3] A.M. Grishin, Mathematical Modeling Forest Fire and New Methods Fighting Them. Publishing House of Tomsk University, Tomsk (1997)

[4] D. Morvan, J.L. Dupuy, Combustion and Flame 138 (2004)

[5] S.V. Patankar, Numerical Heat Transfer and Fluid Flow, Hemisphere Publishing Corporation, New York (1981)

[6] E.N. Valendik, P.M. Mathveev, M.A. Safromov, Large Forest Fires and Fighting with Them. Science, Moscow (in Russian) (1979) 